\title{
BMJ Open Fast track rehabilitation after reversed total shoulder arthroplasty: a protocol for an international multicentre prospective cohort study
}

\author{
Tom van Essen (D) , ${ }^{1}$ Anke Kornuijt, ${ }^{2}$ Lieke Maria Anna de Vries, ${ }^{3}$ Remco Stokman, ${ }^{4}$ \\ Walter van der Weegen, ${ }^{2}$ Rob Bogie, ${ }^{2}$ Robert Jan Hillen, ${ }^{1}$ DA van Kampen ${ }^{5}$
}

To cite: van Essen T, Kornuijt A, de Vries LMA, et al. Fast track rehabilitation after reversed total shoulder arthroplasty: a protocol for an international multicentre prospective cohort study. BMJ Open 2020;10:e034934. doi:10.1136/ bmjopen-2019-034934

\section{- Prepublication history and} additional material for this paper are available online. To view these files, please visit the journal online (http://dx.doi. org/10.1136/bmjopen-2019034934).

Received 11 October 2019 Revised 22 June 2020 Accepted 30 June 2020

\section{Check for updates}

(c) Author(s) (or their employer(s)) 2020. Re-use permitted under CC BY-NC. No commercial re-use. See rights and permissions. Published by BMJ.

${ }^{1}$ Orthopaedic Surgery, Dijklander Ziekenhuis, Purmerend, The Netherlands

${ }^{2}$ Orthopaedic Surgery, Sint Annaziekenhuis, Geldrop, NoordBrabant, The Netherlands ${ }^{3}$ Orthopaedic Surgery, Dijklander Ziekenhuis, Hoorn, The Netherlands

${ }^{4}$ Orthopaedic Surgery, Sint Elisabeth Hospitaal, Willemstad, Curaçao

${ }^{5}$ Orthopedic Surgery and Traumatologie, Dijklander Ziekenhuis, Hoorn, The Netherlands

Correspondence to

Tom van Essen;

tomvanessen11@gmail.com

\section{ABSTRACT}

Introduction The use of reversed total shoulder arthroplasty (rTSA) has increased because of an increasing number of indications for this procedure and by ageing of the population. Usual postoperative care consists of immobilisation of the shoulder for a period of 2-6 weeks to allow healing of the subscapularis tendon and protection of the joint. However, new literature proved that reattachment of the subscapularis tendon is unnecessary. Therefore we hypothesised that immobilisation of the shoulder is not necessary and patients can start safely with mobilisation on the first postoperative day. We expect this fast track protocol to be safe and result in better short-term and long-term functional outcomes.

Methods and analysis In our prospective cohort, we will include at least 75 patients aged 50 years and older indicated for rTSA, with acute fracture treatment as an exclusion criterion. Patients will be selected and operated in three hospitals: two in the Netherlands and one in Curacao.

Patients will visit the outpatient clinic preoperative, at 6 weeks, 3 months and 1 year postoperative. The data that will be collected includes baseline characteristics, reason for surgery, complications and adverse events, patient reported outcomes (Oxford Shoulder Score, EuroQol-5D and Numeric Rating Scale for pain) and range of motion of the shoulder.

All patients will be instructed to use a sling only for 1 day and to follow a progressive physiotherapy schedule for 12 weeks. The primary outcome is the occurrence of complications and adverse events.

Ethics and dissemination The Medical Ethics Committee from the VUmc and Curacao reviewed this study protocol and granted exemption from ethical approval (METC VUmc 2019.111, METC Curacao 2019-02). Study results will be presented at (inter)national conferences and published in peer-reviewed journals.

Trial registration number Netherlands Trial Register (NL7656).

\section{INTRODUCTION}

Reversed total shoulder arthroplasty (rTSA) was introduced by Paul Grammont in the 1980s. ${ }^{1}$ The use of rTSA gained popularity in the treatment of rotator cuff arthropathy.
Strengths and limitations of this study

To our knowledge, the first study to prospectively follow-up early mobilisation after reversed total shoulder arthroplasty, with use of a sling for only 1 day postoperatively.

- Rehabilitation will take place under the supervision of a physiotherapist following a strict protocol.

- Effects will be evaluated using patient-reported outcome measures, clinical outcomes and incidence of complications.

- This study's main limitation is the absence of a control group of patients following the usual rehabilitation protocol, including 6 weeks immobilisation.

- Patients are assessed by the same orthopaedic surgeon preoperatively, perioperatively and postoperatively, increasing the risk of possible measurement bias.

However, since the introduction of rTSA, its' indications have been expanded. Now rTSA is also indicated for patients with massive irreparable rotator cuff tears, for rotator cuff tears with pseudoparalysis, arthritis-inflammatory and non-inflammatory-and for osteoarthritis in the octogenarian. Also patients with an at-risk rotator cuff, fractures or tumours are now indicated for rTSA. ${ }^{2-5}$

Historically, rTSA was considered to be a salvage surgical procedure, used to gain pain relief and restoration of overhead function in low-demand older patients (aged $>70$ years). ${ }^{2}$

With the expansion of the indications for rTSA, the demographic characteristics of patients selected for rTSA have expanded equally. Younger patients and patients with higher activity levels are now indicated for rTSA more often than before ${ }^{67}$ In the USA, up to one-third of all total shoulder arthroplasties is a rTSA. ${ }^{8}$

Despite the gained popularity of rTSA, there is little consensus among published 
rehabilitation guidelines for rTSA. A recent systematic review by Bullock et al showed that the only consensus was that physiotherapy plays an important role in optimising patient outcomes, and that there is a need for high-quality prospective research. ${ }^{9}$ A 2014 Delphi consensus study to create a guideline for preoperative indication and postoperative care after rTSA resulted in disagreement on what the optimal rehabilitation should be and what motions should be allowed. ${ }^{10}$ It also stressed the need for more clinical research. However, today there is still a lack of evidence regarding the postoperative rehabilitation after rTSA. A period of immobilisation ranging from 2 to 6 weeks was described by most studies, but again without prospectively conducted research supporting this advice.

Traditionally patients undergoing rTSA surgery are immobilised for at least 2-6 weeks to protect the operated joint and to allow tissue healing, as described by Boudreau et $a l^{11}$ In the early days, the subscapularis tendon was detached during surgery and reattached to the tuberculum minus, and consequently needed time to heal postoperative. In modern rTSA surgery, reattaching the subscapularis tendon is not mandatory as presented by Friedman et al and Vourazeris et al. ${ }^{12}$ Both studies showed similar outcomes and similar complication rates between reattaching the tendon and not reattaching the tendon. Also, de Boer et al showed no significant differences in a range of motion (ROM) between subscapularis tendon repair or resection. ${ }^{14}$ Without the need to reattach the subscapularis tendon, combined with the stability provided by the modern design of rTSA, patients can start mobilisation directly postoperative. In the study by Denard et al, patients undergoing anatomic total shoulder arthroplasty showed an increased ROM in the first 3 months in patients who were not immobilisated postoperatively compared with usual care. ${ }^{15}$ However, with the need to reattach the subscapularis tendon in anatomic TSA to the lower tuberosity in this type of surgery, the healing rate of the lower tuberosity decreased significantly from $96 \%$ to $81 \%$. This decreased healing rate will not pose a problem in our study, since we will not reattach the subscapularis tendon for the rTSA, as reattachment is not mandatory.

The study of Hagen investigated early mobilisation versus 6 weeks of immobilisation and concluded it was safe to start mobilisation early. ${ }^{16}$ This study reported similar results between early mobilisation and 6 weeks of immobilisation on the short term and long term. However, the study started mobilisation 1 week postoperative, we start mobilisation directly postoperative. Therefore, we expect patients will have better outcomes on the short term and will be independent quicker.

Without immobilisation of the arm and with patients starting to mobilise immediately postoperatively, guided by a strict fast track protocol, we expect a faster postoperative recovery, an increased shoulder function and improved self-reported functional outcome on short term without an increase in complications and adverse events. We also expect patients to be less dependent on the care
Box 1 Inclusion and exclusion criteria

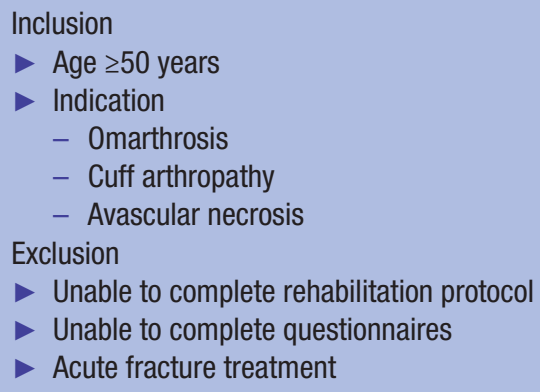

of a district nurse or family members, thereby reducing healthcare costs.

\section{METHODS AND ANALYSIS}

This study is an international multicentre prospective cohort study.

\section{Study setting}

The study will be performed in two peripheral hospitals in the Netherlands (Dijklander hospital in Hoorn and Purmerend and St Anna hospital in Geldrop) and one hospital in Curacao (St Elisabeth Hospital in Willemstad). The population will be drawn from patients presented to the outpatient orthopaedic clinic with shoulder complaints and selected for rTSA.

All patients will undergo elective surgery; no emergency patients will be included.

\section{Recruitment}

Patients will be selected in the outpatient clinics of participating hospitals by an orthopaedic surgeon (DAvK, RJH, RB, and RS). Patients will be considered for participation if they fulfil all inclusion criteria and none of the exclusion criteria (box 1). Patients undergoing acute fracture treatment are excluded because reattachment of all available rotator cuff tendons and the greater tubercle is mandatory for good postoperative function, as demonstrated by Obert et al. . $^{17}$

The research staff will follow good clinical practice and ensure that the participants have a complete understanding of the study before signing the informed consent. Patients will have a sufficient amount of time to consider participation. If a patient is eligible, he or she will be included after signing the informed consent. In online supplementary appendix 1, patient information form and informed consent form is added. Recruitment of patients started on 1 March 2019.

Baseline characteristics will be collected and preoperative evaluation of the shoulder function will be completed. The preoperative shoulder ROM will be measured by the examiner, which is the orthopaedic surgeon at the outpatient clinic, in abduction, forward flexion, external rotation and internal rotation following a standardised protocol. The participant is asked to 
complete the following questionnaires: the Oxford Shoulder Score (OSS), the EuroQol-5D (EQ-5D, quality of life) and Numeric Rating Scale (NRS) for pain in rest and during activity. ${ }^{18}{ }^{19}$ Questionnaires will be sent by email if possible, otherwise, hard copies will be handed out during outpatient clinic visits.

\section{Adverse events and complications}

We will gather all information on all adverse events and complications. Adverse events following rTSA can be very diverse and are often patients specific. Described complications following rTSA are dislocation, periprosthetic fractures, acromion stress fractures and glenosphere migration. ${ }^{16}$ Also a total complication rate of $20 \%$ is described by Clarck. ${ }^{20}$ We will look into every adverse event and complication in detail to investigate if this is a result of our fast track protocol.

\section{Range of motion}

The ROM will be measured using a standardised protocol. Abduction and forward flexion will be measured using a goniometer. External rotation will be estimated with the shoulder in $0^{\circ}$ abduction and the elbow in $90^{\circ}$ flexion. Internal rotation will be measured by the method described in the Constant score, with the palm of the hand as target. ${ }^{21}$

\section{Oxford Shoulder Score}

The OSS is a questionnaire on shoulder function. It is a 12 questions questionnaire scoring $1-5$ each. The best possible score is 12, which corresponds with an excellent shoulder function with no pain. The worst possible score is 60 , which corresponds with a very poor shoulder function with unbearable pain. ${ }^{19}$ We will use the validated Dutch version of the OSS . ${ }^{22}$

\section{EuroQol 5-D}

The EQ-5D questionnaire is standardised questionnaire to assess the generic health status of an individual. It consists of five domains (mobility, self-care, usual activities, pain/ discomfort and anxiety/depression) which are scored
$1-3$, and it asks the participant to score their health on a visual analogue scale. It provides an assessment of the preoperative and postoperative quality of life. ${ }^{18}$ We will use the validated Dutch version of the EQ-5D.

\section{Numeric Rating Scale for pain}

The NRS gives patients the possibility to score the pain on a scale of $0-10$, in which a score of 0 means no pain and 10 means the worst possible pain. We will ask for NRS at rest and during activity.

Participants will return for postoperative follow-up according to the standard protocol. The follow-up will take place 6 weeks ( \pm 1 week), 3 months ( \pm 2 weeks) and 12 months ( \pm 4 weeks) postoperative (see table 1 ). At each visit, the shoulder function will be registered and patients will complete the same questionnaires as preoperatively. One additional questionnaire, an adjusted version of the Tic-P CIPRUS questionnaire, is added 3 months postoperatively to evaluate the healthcare costs made.

\section{Tic-P CIPRUS}

This is a questionnaire measuring the use of healthcare direct postoperatively from a societal perspective. It consists of different domains which allow for the measurement of medical costs and productivity losses in work or volunteer work. This is quantified by the time spent or lost on different activities, for example, rehabilitation or absence at work.

\section{Procedure}

All surgeries will be performed in a standard beach chair position using a deltopectoral approach. In all procedures, the subscapularis tendon will be released. Stability of the arthroplasty will be tested intraoperatively. The Dijklander hospital uses the Equinoxe reverse shoulder prosthesis by Exactech. The St Anna Hospital and the St Elisabeth Hospital use the Comprehensive Reverse shoulder prosthesis by Zimmer Biomet. ${ }^{23} 24$

Patients will be hospitalised on the day of surgery and discharged between 0 and 2 days postoperative

\begin{tabular}{|c|c|c|c|c|c|}
\hline & Preoperative & Perioperative & $\begin{array}{l}6 \text { weeks } \\
\text { postoperative }\end{array}$ & $\begin{array}{l}3 \text { months } \\
\text { postoperative }\end{array}$ & $\begin{array}{l}12 \text { months } \\
\text { postoperative }\end{array}$ \\
\hline Baseline characteristics & $x$ & & & & \\
\hline Range of motion & $x$ & & $\mathrm{x}$ & $\mathrm{x}$ & $\mathrm{x}$ \\
\hline Perioperative record & & $x$ & & & \\
\hline Oss & $x$ & & $x$ & $x$ & $x$ \\
\hline EQ-5D & $x$ & & $x$ & $x$ & $x$ \\
\hline NRS pain & $x$ & & $x$ & $x$ & $x$ \\
\hline Tic-P CIPRUS & & & & $x$ & \\
\hline $\begin{array}{l}\text { Complications and } \\
\text { readmissions }\end{array}$ & & $\mathrm{x}$ & $\mathrm{x}$ & $\mathrm{x}$ & $\mathrm{X}$ \\
\hline
\end{tabular}

EQ-5D, EuroQol-5D; NRS, Numeric Rating Scale; OSS, Oxford Shoulder Score. 
Table 2 Physiotherapy protocol

\begin{tabular}{|c|c|c|}
\hline Phase & Physiotherapy & Aim \\
\hline I (0-1 week) & $\begin{array}{l}\text { Start with physiotherapy first day postoperative } \\
\text { Focus on } \\
\text { 1. Elbow function, wrist function and hand function } \\
\text { Scapula setting } \\
\text { Guided active movement of the shoulder (max } 90^{\circ} \text { forward } \\
\text { flexion): raise arms, swing, external rotation with a stick (in } \\
\text { supine position) }\end{array}$ & $\begin{array}{l}\text { 1. Decrease swelling and pain } \\
\text { 2. End of phase I } \\
\text { At least } 45^{\circ} \text { forward flexion } \\
\text { At least } 45^{\circ} \text { abduction } \\
\text { Passive, if possible active }\end{array}$ \\
\hline II (2-4 weeks) & $\begin{array}{l}\text { Start mobilising the glenohumeral joint, no ROM restrictions. } \\
\text { Forward flexion, abduction and external rotation guided by } \\
\text { pain. } \\
\text { Focus on } \\
\text { Extend exercises of phase I } \\
\text { - Isometric exercises of musculus deltoideus } \\
\text { Guided active movement of musculus deltoideus }\end{array}$ & $\begin{array}{l}\text { 1. Decrease swelling and pain } \\
\text { 2. Recovery of ROM and muscle } \\
\text { activation } \\
\text { 3. End of phase II } \\
\text { At least } 70^{\circ} \text { forward flexion } \\
\text { - At least } 70^{\circ} \text { abduction } \\
\text { - Passive, if possible active }\end{array}$ \\
\hline III (4-6 weeks) & $\begin{array}{l}\text { Mobilising glenohumeral joint. } \\
\text { Retrieve ROM and start muscle activation. } \\
\text { Focus on } \\
\text { Pulley and bench slides (later on wall slides) } \\
\text { Strengthen musculus deltoideus, in different body positions } \\
\text { Strengthen scapula-thoracal musculature } \\
\text { If present: strengthen the rotator cuff }\end{array}$ & $\begin{array}{l}\text { 1. Retrieve function and ROM. } \\
\text { 2. End of phase III } \\
\text { At least } 90^{\circ} \text { forward flexion } \\
\text { At least } 80^{\circ} \text { abduction } \\
\text { Passive, if possible active }\end{array}$ \\
\hline IV (7-12 weeks) & $\begin{array}{l}\text { Mobilising glenohumeral joint, reaching for preoperative values } \\
\text { or contralateral side. } \\
\text { Focus on } \\
\text { - Strengthen musculus deltoideus } \\
\text { Strengthen scapula-thoracal musculature } \\
\text { - Strengthen all other shoulder musculature } \\
\text { ADL training }\end{array}$ & $\begin{array}{l}\text { 1. Recovery to optimal ROM, strength } \\
\text { and endurance. } \\
\text { 2. End of phase IV } \\
>>90^{\circ} \text { forward flexion } \\
>80^{\circ} \text { abduction } \\
- \text { Passive, if possible active } \\
\text { 3. Back to work and participate in } \\
\text { activities of daily life. }\end{array}$ \\
\hline V (>12 weeks) & \multicolumn{2}{|c|}{ Improve ROM and optimise strength, start participating in ADL/work/sports. } \\
\hline Living rules & \multicolumn{2}{|c|}{$\begin{array}{l}\text { Sling first day postoperative and if needed during the first } 2 \text { weeks, during the night not needed } \\
\text { Allowed to sleep on the affected side if the pain is tolerable } \\
\text { Lifting close to the body }\end{array}$} \\
\hline
\end{tabular}

$A D L$, activities of daily living.

following protocol. The rehabilitation will start the first day postoperatively under supervision of a study-trained physiotherapist.

Preoperatively the patients will have an ultrasoundguided interscalene pain block and are therefore unable to use the arm directly postoperative. The rehabilitation will follow a strict protocol especially designed for postoperative care after rTSA and in concordance with physiotherapists (see table 2). At discharge, patients receive a written protocol for the treating physiotherapist, which includes a detailed description of the rehabilitation postrTSA. Research staff will contact treating physiotherapists to instruct them on the use of the new fast track protocol.
Patients' compliance will be checked during outpatient clinic visits.

\section{Outcome measures}

The primary study parameter is the occurrence of complications or adverse events.

We will investigate differences in OSS, shoulder ROM, NRS pain scores, quality of life (EQ-5D) between preoperative and 6 weeks, 3 months and 1 year postoperative as secondary parameters. At 3 months postoperative the society costs, including healthcare, patient and family costs and lost productivity costs, will be measured using the Tic-P CIPRUS questionnaire. 
Besides the lack of immobilisation and the structured rehabilitation protocol, all provided treatments and follow-up are standard of care and no additional or divergent medication, interventions or investigational medical devices will be applied in this study. After 6 months an interim analysis will be performed to determine safety.

\section{Data analysis}

As a primary analysis the occurrence of complications and adverse events will be collected and divided in subgroups. Means will be calculated.

As secondary analyses the difference in OSS between preoperative and 6 weeks and 3 months and 12 months postoperative will be calculated. Subsequently, the difference in NRS pain, in EQ-5D and shoulder ROM between preoperative and 6 weeks, 3 months and 12 months postoperative will be calculated.

With the results of the Tic-P CIPRUS, we will analyse healthcare costs.

All adverse events and complications will be registered and presented in number and severity.

Assuming normal distributions, means will be calculated and a mean difference will be calculated for all outcomes. All means and mean differences will be supplied with an SD. For all differences, a Student's t-test will be performed to test for significance. If the distribution does not show normality a Wilcoxon signed-rank test will be used to calculate differences, medians will be calculated accompanied with an IQR.

\section{Sample size estimation}

We decided to include all patients meeting the criteria for 1 year and did not perform a power analysis. We estimate to include at least 50 patients in 1 year, but we will continue including patients until we have reached 75 patients. This number correlates with similar studies evaluating the effect of rTSA. For example the study by Sadoghi et al which evaluated the infraglenoidal scapular notching, which included 60 patients. ${ }^{25}$ Or the study by de Boer $e t a l$ which evaluated the influence of the subscapularis tendon on the ROM in rTSA, which included 65 patients. ${ }^{14}$

\section{Patients and public involvement}

Patients and public were not involved in the design of this study and will not be involved in the recruitment to or conduct of this study. However, study results will be disseminated to all study participants by sending them an (e) mail with our study results, phrased without medical jargon

\section{ETHICS AND DISSEMINATION}

The Medical Ethics Committee from the VUmc and Curacao reviewed this study protocol and granted exemption from ethical approval (METC VUmc 2019.111, METC Curacao 2019-02).
Participants will not experience any undue hardship, expenses or time commitments because the questionnaires will be sent digitally by email or otherwise carried out during the participants' routine preoperative and postoperative visits.

Informed consent will be asked from all participants. The study ends when the patient is 1 year postoperative. The acquired digital and non-digital data will be kept for a minimum of 15 years following hospital research protocol. Patient information will be coded and privacy aspects of the database will be guarded according to Dutch legislation. The key-database to encode the database will be locked by a password that is only open for the participating investigators.

Our study results will be presented at (inter)national conferences and published in peer-reviewed journals.

\section{DISCUSSION}

To our knowledge, this is the first prospective study evaluating immediate mobilisation the first day postoperative after reversed total shoulder arthroplasty without the need to wear a sling for 6 weeks. We designed a strict protocol with different phases, each phase, including guidelines on what to do and what to expect (see table 2). The protocol was designed in close collaboration with experienced physiotherapists. We expect that direct mobilisation after rTSA is safe and will be of great value, just as in total knee and total hip arthroplasty.

Without the need to immobilise the shoulder for a period of up to 6 weeks, we expect the patients to recover faster and therefore be more self-reliant and less dependent on the care of partner or district nurse. This will result in fewer healthcare costs and also we expect (partners of) patients can return to work faster.

A limitation of our study is that patients will visit the outpatient clinic with the same physician who performed surgery, creating a possible measurement bias for our secondary outcome: ROM. To minimise the risk of bias, we developed a standardised protocol to measure shoulder ROM, which will be strictly followed by all physicians.

We expect that the prospective follow-up of our new protocol will provide new insights and will change the current postoperative care after rTSA and prove to be a safe option. The use of different brands of rTSA increases the external validity.

Contributors TvE, DAvK and LMAdV initiated the study. TvE, DAvK, LMAdV, AK and WvdW involved in study design and protocol design. RB, RJH, RS and DAvK orthopaedic surgeons, including the patients at the out patient clinic and performing surgery.

Funding The authors have not declared a specific grant for this research from any funding agency in the public, commercial or not-for-profit sectors.

Competing interests van Kampen and Hillen have a consultancy contract with Exactech Company. This study was not sponsored by Exactech. Bogie has a consultancy contract with Zimmer Biomet. This study was not sponsored by Zimmer Biomet.

Patient consent for publication Not required.

Provenance and peer review Not commissioned; externally peer reviewed. 
Open access This is an open access article distributed in accordance with the Creative Commons Attribution Non Commercial (CC BY-NC 4.0) license, which permits others to distribute, remix, adapt, build upon this work non-commercially, and license their derivative works on different terms, provided the original work is properly cited, appropriate credit is given, any changes made indicated, and the use is non-commercial. See: http://creativecommons.org/licenses/by-nc/4.0/.

ORCID iD

Tom van Essen http://orcid.org/0000-0001-9256-9269

\section{REFERENCES}

1 Grammont P, Trouilloud P, Laffay J, et al. Concept study and realization of a new total shoulder prosthesis. Rhumatologie 1987;39:407-18.

2 Simovitch RW, Gerard BK, Brees JA, et al. Outcomes of reverse total shoulder arthroplasty in a senior athletic population. J Shoulder Elbow Surg 2015;24:1481-5.

3 Guery J, Favard L, Sirveaux F, et al. Reverse total shoulder arthroplasty. survivorship analysis of eighty replacements followed for five to ten years. J Bone Joint Surg Am 2006;88:1742-7.

4 Favard L, Levigne C, Nerot C, et al. Reverse prostheses in arthropathies with cuff tear: are survivorship and function maintained over time? Clin Orthop Relat Res 2011;469:2469-75.

5 Boileau P, Watkinson D, Hatzidakis AM, et al. Neer award 2005: the Grammont reverse shoulder prosthesis: results in cuff tear arthritis, fracture sequelae, and revision arthroplasty. J Shoulder Elbow Surg 2006;15:527-40.

6 Ek ETH, Neukom L, Catanzaro S, et al. Reverse total shoulder arthroplasty for massive irreparable rotator cuff tears in patients younger than 65 years old: results after five to fifteen years. $J$ Shoulder Elbow Surg 2013;22:1199-208.

7 Sershon RA, Van Thiel GS, Lin EC, et al. Clinical outcomes of reverse total shoulder arthroplasty in patients aged younger than 60 years. $J$ Shoulder Elbow Surg 2014;23:395-400.

8 Schairer WW, Nwachukwu BU, Lyman S, et al. National utilization of reverse total shoulder arthroplasty in the United States. J Shoulder Elbow Surg 2015;24:91-7.

9 Bullock GS, Garrigues GE, Ledbetter L, et al. A systematic review of proposed rehabilitation guidelines following anatomic and reverse shoulder arthroplasty. J Orthop Sports Phys Ther 2019;49:337-46.

10 Kwaees TA, Charalambous CP. Reverse shoulder arthroplasty-minimum age for surgery, postoperative rehabilitation and long term restrictions. A delphi consensus study. Ortop Traumatol Rehabil 2014;16:435-9.
11 Boudreau S, Boudreau ED, Higgins LD, et al. Rehabilitation following reverse total shoulder arthroplasty. J Orthop Sports Phys Ther 2007;37:734-43.

12 Friedman RJ, Flurin P-H, Wright TW, et al. Comparison of reverse total shoulder arthroplasty outcomes with and without subscapularis repair. J Shoulder Elbow Surg 2017;26:662-8.

13 Vourazeris JD, Wright TW, Struk AM, et al. Primary reverse total shoulder arthroplasty outcomes in patients with subscapularis repair versus tenotomy. J Shoulder Elbow Surg 2017;26:450-7.

14 de Boer FA, van Kampen PM, Huijsmans PE. The influence of subscapularis tendon reattachment on range of motion in reversed shoulder arthroplasty: a clinical study. Musculoskelet Surg 2016;100:121-6.

15 Denard PJ, Lädermann A. Immediate versus delayed passive range of motion following total shoulder arthroplasty. J Shoulder Elbow Surg 2016;25:1918-24.

16 Hagen MS, Allahabadi S, Zhang AL, et al. A randomized singleblinded trial of early rehabilitation versus immobilization after reverse total shoulder arthroplasty. J Shoulder Elbow Surg 2020;29:442-50.

17 Obert L, Saadnia R, Tournier C, et al. Four-part fractures treated with a reversed total shoulder prosthesis: prospective and retrospective multicenter study. results and complications. Orthop Traumatol Surg Res 2016;102:279-85.

18 Rabin R, de Charro F. EQ-5D: a measure of health status from the EuroQol group. Ann Med 2001;33:337-43.

19 Dawson J, Fitzpatrick R, Carr A. Questionnaire on the perceptions of patients about shoulder surgery. J Bone Joint Surg Br 1996;78:593-600.

20 Clark JC, Ritchie J, Song FS, et al. Complication rates, dislocation, pain, and postoperative range of motion after reverse shoulder arthroplasty in patients with and without repair of the subscapularis. J Shoulder Elbow Surg 2012;21:36-41.

21 Constant CR, Murley AH. A clinical method of functional assessment of the shoulder. Clin Orthop Relat Res 1987;214:160-4.

22 Berendes T, Pilot P, Willems J, et al. Validation of the Dutch version of the Oxford shoulder score. J Shoulder Elbow Surg 2010;19:829-36.

23 Exactech. Equinoxe strength in numbers. Available: https://www. exac.com/extremities/equinoxe-strength-in-numbers/ [Accessed 31 Jul 2019].

24 ZimmerBiomet. Comprehensive reverse shoulder system. Available: https://www.zimmerbiomet.com/medical-professionals/shoulder/ product/comprehensive-reverse-shoulder-system.html [Accessed 31 Jul 2019].

25 Sadoghi P, Leithner A, Vavken P, et al. Infraglenoidal scapular notching in reverse total shoulder replacement: a prospective series of 60 cases and systematic review of the literature. BMC Musculoskelet Disord 2011;12:101. 